\title{
A 3-D Well-Differentiated Model of Pediatric Bronchial Epithelium Demonstrates Unstimulated Morphological Differences Between Asthmatic and Nonasthmatic Cells
}

\author{
JEREMY PARKER, SEVERINE SARLANG, SURENDRAN THAVAGNANAM, GRACE WILLIAMSON, DARA O'DONOGHUE, \\ REMI VILLENAVE, ULTAN POWER, MICHAEL SHIELDS, LIAM HEANEY, AND GRZEGORZ SKIBINSKI
}

Centre for Infection and Immunity [J.P., S.S., S.T., G.W., D.O., R.V., U.P., M.S., L.H.] and School of Medicine, Dentistry and Biomedical Science [G.S.], Queen's University Belfast, Belfast, BT12 6BN, Northern Ireland; Royal Belfast Hospital for Sick Children [M.S.], Belfast

Trust, Belfast, BT12 6BE, Northern Ireland

\begin{abstract}
There is a need for reproducible and effective models of pediatric bronchial epithelium to study disease states such as asthma. We aimed to develop, characterize, and differentiate an effective, an efficient, and a reliable three-dimensional model of pediatric bronchial epithelium to test the hypothesis that children with asthma differ in their epithelial morphologic phenotype when compared with nonasthmatic children. Primary cell cultures from both asthmatic and nonasthmatic children were grown and differentiated at the air-liquid interface for $28 \mathrm{~d}$. Tight junction formation, MUC5AC secretion, IL-8, IL-6, prostaglandin E2 production, and the percentage of goblet and ciliated cells in culture were assessed. Well-differentiated, multilayered, columnar epithelium containing both ciliated and goblet cells from asthmatic and nonasthmatic subjects were generated. All cultures demonstrated tight junction formation at the apical surface and exhibited mucus production and secretion. Asthmatic and nonasthmatic cultures secreted similar quantities of IL-8, IL-6, and prostaglandin E2. Cultures developed from asthmatic children contained considerably more goblet cells and fewer ciliated cells compared with those from nonasthmatic children. A well-differentiated model of pediatric epithelium has been developed that will be useful for more in vivo like study of the mechanisms at play during asthma. (Pediatr Res 67: 17-22, 2010)
\end{abstract}

$\mathrm{T}$ he bronchial epithelium is the interface between the airways and a hostile environment. Rather than acting as a passive barrier, it has been increasingly recognized as having a central role in the immune response to airborne irritants, allergens, and microorganisms (1). In healthy individuals, the bronchial epithelium is a columnar, pseudostratified layer, containing ciliated, goblet, and basal cells. In diseases such as asthma, the bronchial epithelium plays an important role in disease pathogenesis (2). In asthma, it is widely recognized that the disease process begins at an early stage in life (3-5) and progresses into adulthood. Therefore, investigating the

Received April 27, 2009; accepted September 6, 2009.

Correspondence: Grzegorz Skibinski, Ph.D., Respiratory Medicine Research Cluster, School of Medicine, Dentistry and Biomedical Science, Queen's University Belfast, Microbiology Building, Grosvenor Road Belfast, Northern Ireland BT12 6BN, United Kingdom; e-mail: g.skibinski@qub.ac.uk

Supported by Northern Ireland Chest Heart and Stroke Association Grant 200546 and Royal Belfast Hospital for Sick Children.

J.P. and S.S. contributed equally to this study. airway epithelium in childhood is important to our understanding of the processes involved in disease evolution.

We have previously described a safe and effective nonbronchoscopic sampling method for obtaining bronchial epithelial cells from pediatric subjects (6). There is limited data on epithelial function in childhood asthma. One study detailed biochemical and functional differences between bronchial epithelial cells from children with and without asthma using submerged cultures (7). Although insightful, the study did not comprehensively represent the bronchial epithelium in vivo because the monolayer cultures they used lack goblet and ciliated cells that result from differentiation, a naturally occurring process in vivo. We believe that the development of a well-differentiated model of pediatric bronchial epithelium will provide a more detailed and realistic culture model for assessing the differences that are known to exist in vivo.

Culturing primary bronchial epithelial cells (PBECs) in an air-liquid interface (ALI) model was shown to produce a pseudostratified epithelium with differentiated cells $(8,9)$ and such cultures are more representative of in situ bronchial epithelium than monolayer cultures. These cultures, derived from both animal and adult human cells, have been instrumental in increasing our knowledge of epithelial cell physiology (10) and responses to insults such as bacterial and viral infection $(11,12)$. The establishment of a novel welldifferentiated primary bronchial epithelial cell (WD-PBEC) model using epithelial cells from children would therefore be useful in addressing these issues in childhood asthma.

Our initial aim for this study was to generate robust protocols for the reliable generation of authentic, pseudostratified pediatric WD-PBECs. Subsequently, we exploited these protocols to compare and contrast the differentiation phenotypes of bronchial epithelial cells derived from asthmatic and nonasthmatic children. We hypothesized that differentiated bronchial epithelial cells from children with asthma would express higher numbers of mucus-secreting goblet cells compared with nonasthmatic controls. We also investigated whether there were functional differences, specifically IL-8, IL-6, and

Abbreviations: ALI, air-liquid interface; PFA, paraformaldehyde; TEER, transepithelial electrical resistance; TEM, transmission electron microscopy 
Table 1. Information on asthmatic and nonasthmatic subjects

\begin{tabular}{lrll}
\hline Sex $(\mathrm{M} / \mathrm{F})$ & Age $(\mathrm{y})$ & Clinical treatment & \multicolumn{1}{c}{ Atopic history } \\
\hline $\begin{array}{c}\text { Asthmatic } \\
\text { subjects }\end{array}$ & & & \\
$\mathrm{M}$ & 6 & ICS, LAB2A, LTA & Hayfever \\
$\mathrm{M}$ & 7 & ICS, LAB2A, LTA & Eczema \\
$\mathrm{M}$ & 7 & ICS, LAB2A, LTA, & $\begin{array}{l}\text { Eczema, hayfever, } \\
\text { antihistamine }\end{array}$ \\
& \multicolumn{4}{c}{ food allergy } \\
$\mathrm{M}$ & 8 & ICS & \\
$\mathrm{M}$ & 8 & ICS, LAB2A & Eczema, hayfever \\
$\mathrm{F}$ & 10 & ICS, LAB2A & \\
$\mathrm{M}$ & 9 & ICS, LAB2A & Hayfever \\
Nonasthmatic & & & \\
subjects & & & \\
$\mathrm{M}$ & 10 & None & None \\
$\mathrm{M}$ & 7 & None & None \\
$\mathrm{M}$ & 10 & None & None \\
$\mathrm{F}$ & 8 & None & None \\
$\mathrm{F}$ & 12 & None & None \\
$\mathrm{M}$ & 10 & None & Hayfever \\
$\mathrm{M}$ & 7 & None & Eczema, hayfever \\
$\mathrm{F}$ & 4 & None & Eczema, food allergy \\
$\mathrm{F}$ & 4 & None & Eczema, food allergy \\
\hline
\end{tabular}

ICS, inhaled corticosteroids; LAB2A, long-acting beta2 agonist; LTA, leukotriene antagonist.

prostaglandin E2 (PGE2) secretion between asthmatic and nonasthmatic children as previously reported (7).

\section{METHODS}

Subjects. Samples were obtained from seven asthmatic and nine nonasthmatic children between the ages of 4 and 12 y attending the Royal Belfast Hospital for Sick Children for elective surgical procedures. Table 1 provides information on the asthmatic and nonasthmatic subjects, including their sex, age, treatment, and atopic status. Nonasthmatic subjects had no history of persistent respiratory symptoms. Written parental consent was obtained before sampling as well as permission from children who understood the nature of the procedure. Detailed questionnaires were administered by physicians (13). The study was approved by the Research Ethics Committee of Queen's University Belfast.

Isolation of primary pediatric bronchial epithelial cells. Nonbronchoscopic bronchial brushings were obtained from asthmatic children $(n=7)$ and nonasthmatic children $(n=9)$, as described by Doherty et al. (6). Briefly, the bronchial brushing was performed by inserting a bronchial cytology brush through the endotracheal tube and wedging the tip in a peripheral bronchus. At this point, the brush and sheath are withdrawn $2 \mathrm{~cm}$, followed by the brush being advanced $2 \mathrm{~cm}$ and gentle brushing performed. The brush was then withdrawn before removal. PBECs were cultured using methods developed in our laboratory, which resulted in homogenous cultures of basal epithelial cells (6).

Collagen coating of flasks and transwell inserts. Flasks and transwell inserts were coated using PureCol bovine purified collagen (Inamed Biomaterials, Fremont, CA). Collagen working solution was made using a 1:100 dilution of PureCol $(3 \mathrm{mg} / \mathrm{mL})$ in $\mathrm{ddH}_{2} \mathrm{O}$ giving a working concentration of $0.03 \mathrm{mg} / \mathrm{mL}$. Two hundred microliters of working collagen solution was added to each transwell $\left(0.005 \mathrm{mg} / \mathrm{cm}^{2}\right)$ and was allowed to air-dry in a laminar flow hood overnight before being exposed to UV radiation for $20 \mathrm{~min}$ the following morning.

ALI cultures for establishment of well-differentiated mucociliary epithelium. ALI cultures were grown in line with methods previously described by Gray et al.(8) and Fulcher et al.(9). PBECs were grown initially on collagencoated T10 flasks and grown until $80 \%$ confluency, then passed to collagen coated T75 flasks for expansion. Briefly, once PBECs grown in growth factor supplemented BEGM (Promocell, Heidelberg, Germany) had reached $\sim 80 \%$ confluency in T75 flasks, cells were detached, trypan blue assessed for viability, counted, and seeded at a cell density of $0.8 \times 10^{5}$ viable cells onto collagen-coated 12-mm Transwell-Clear inserts $0.4-\mu \mathrm{m}$ pore size (Corning Life Sciences, MA). As described by Gray et al. (8), cells were cultured on inserts in an ALI medium consisting of a 50:50 mixture of AEGM (Promocell, Heidelberg, Germany) and DMEM (Invitrogen Ltd, Paisley, UK) supplemented with bovine pituitary extract $(52 \mu \mathrm{g} / \mathrm{mL})$, epidermal growth factor
$(0.5 \mathrm{ng} / \mathrm{mL})$, insulin $(5 \mu \mathrm{g} / \mathrm{mL})$, hydrocortisone $(0.5 \mu \mathrm{g} / \mathrm{mL})$, epinephrine $(0.5$ $\mu \mathrm{g} / \mathrm{mL})$, transferrin $(10 \mu \mathrm{g} / \mathrm{mL})$, BSA $(1.5 \mu \mathrm{g} / \mathrm{mL})$, penicillin/streptomycin $(100 \mathrm{IU} / \mathrm{mL} / 100 \mu \mathrm{g} / \mathrm{mL})$, and retinoic acid $(50 \mathrm{nM})$. Cells were grown under submerged conditions until confluent. At this stage, an ALI was created by removing the medium from the apical side to promote mucociliary differentiation. This was considered to be d 0 of ALI culture and the start of our experimental period. The cells were fed only from the basolateral layer every other day with ALI medium and the apical side was washed weekly with PBS at $37^{\circ} \mathrm{C}$. Apical washings were kept and stored at $-80^{\circ} \mathrm{C}$ for later analysis. All cells from subjects used in this study were grown at ALI at passage 3 . Cultures were grown for $28 \mathrm{~d}$ at ALI to ensure full differentiation as assessed by the presence of beating cilia and mucus production observed as visible mucus build up on the apical surface of the cultures.

Transepithelial electrical resistance. Transepithelial electrical resistance (TEER) was measured on $\mathrm{d} 7,14,21$, and 28 to ensure the formation and integrity of tight junctions between cells in the epithelium using an EVOM meter (World Precision Instruments, FL). Before measurement, the apical layer of the cultures was washed with PBS and then DMEM was added to the apical and basolateral side to equilibrate the cultures. Measurements were performed three times per well and a mean resistance calculated. Values were then corrected for the blank resistance (membrane with no cells) and the surface area.

MUC5AC ELISA. On d 7, 14, 21, and 28 of ALI culture, the apical layer of the cells were washed with $500 \mu \mathrm{L}$ PBS, to remove mucus secretions, and stored at $-80^{\circ} \mathrm{C}$ for ELISA analysis at a later date. The method used was adapted from Takeyama et al.(14). Briefly, apical washings were diluted (1:5) in carbonatebicarbonate coating buffer (Sigma Chemical Co., Poole, Dorset, UK) and incubated overnight at $37^{\circ} \mathrm{C}$. After washing in PBS, a 1:200 dilution of MUC5AC mouse MAb (Abcam, Cambridge, UK) was incubated for $1 \mathrm{~h}$ at room temperature. After washing in PBS, a 1:10,000 dilution of goat polyclonal anti-mouse IgG antibody conjugated to HRP (Novus Biologicals, Littleton) was incubated for $1 \mathrm{~h}$ at room temperature. After washing, $3^{\prime} 5^{\prime}$ trimethylbenzoate was added to each well and incubated in the dark for $15 \mathrm{~min}$ to allow color to develop. Stop solution $\left(2 \mathrm{~N} \mathrm{H}_{2} \mathrm{SO}_{4}\right)$ was added and absorbance measured on a plate reader. Results were expressed as absorbance $(450 \mathrm{~nm})$.

RNA extraction and RT-PCR for MUC5AC mRNA. WD-PBECs harvested for RNA extraction were detached by trypsinization from the membrane and kept in RNAlater (Applied Biosystems, Warrington, UK) to stabilize their cellular RNA profile. Total RNA was extracted from stabilized cells using RNeasy Mini kit (Qiagen, Crawley, UK) according to the manufacturer's instructions and quantified on a spectrophotometer. Detection of the MUC5AC gene in the cultures was carried out by standard RT-PCR, as previously described (15). RT-PCR reactions were performed using SuperArray ReactionReady First Strand cDNA Synthesis Kit and HotStart "Sweet" PCR mastermix (Tebu-bio, Peterborough, UK) according to the manufacturer's recommendations. Primers were designed according to the following description:

Forward 5' TCC TTT CGT GTT GTC ACC GA 3' localization on cDNA: $2874 \mathrm{bp}$

Reverse $5^{\prime}$ TCT TGA TGG CCT TGG AGC A $3^{\prime}$ localization on cDNA: $2943 \mathrm{bp}$

Equal loading of cDNA was evaluated by assessing the expression of house keeping gene GAPDH. After RT-PCR, samples were loaded on a $2 \%$ agarose gel and visualized by ethidium bromide.

IL-6 and IL-8 ELISA. Cytokine concentrations were measured from aliquots of basolateral culture medium taken on d 28 of culture using commercial IL-6 ELISA kits from Sanquin (Amsterdam, The Netherlands) and IL-8 ELISA kits from R \& D Systems Europe (Abingdon, UK).

PGE2 EIA. PGE2 concentrations were measured from aliquots of basolateral culture medium taken on d 28 of culture using a commercial enzyme immunoassay (EIA) kit from IDS Ltd (Tyne \& Wear, UK).

Immunohistochemistry and Immunocytochemistry for goblet and ciliated cell markers. Selected intact cultures grown at the ALI were washed with PBS, fixed in 4\% paraformaldehyde (PFA), dehydrated, and paraffin embedded. Five-micrometer sections were cut according to standard procedure and stained with hematoxylin and eosin. Cytospin slides were also made for selected cultures. Goblet cells and ciliated cells were detected using a 1:100 dilution of mouse MAb against MUC5AC (Abcam, Cambridge, UK) and a 1:700 dilution of mouse MAb against acetylated alpha tubulin (Abcam, Cambridge, UK), respectively, for $2 \mathrm{~h}$ at room temperature. Specific binding was detected by a micropolymer of active peroxidase coupled to anti-mouse IgG secondary antibodies (ImmPress Universal Reagent, Vector Laboratories, Burlingame, CA).

These sections were stained using DAB peroxidase substrate kit (Vector Laboratories, Peterborough, UK). Slides ( $n=4 /$ subject/treatment $)$ were mounted using DPX (D\&H, Belfast, Northern Ireland) and viewed under a 
light microscope. Negative control slides were also prepared in the same fashion with the primary antibody being omitted from the incubation buffer to demonstrate specificity. The numbers of positive cells for each stain are represented as a mean \% of 1000 cells counted per slide.

Immunofluorescence and confocal microscopy. Selected cultures were fixed in 4\% PFA and washed three times in PBS. Cells were permeabilized using 0.2\% Triton-X 100 (Sigma Chemical Co.-Aldrich, Dorset, UK) for $1 \mathrm{~h}$ followed by three washes in PBS. Cells were then exposed to either a 1:100 dilution of rabbit anti-MUC5AC primary antibody (goblet cells) (Santa Cruz Biotechnology Inc., CA), a 1:100 dilution of mouse anti-MUC5AC primary antibody (goblet cells) (Abcam, Cambridge, UK), a 1:100 dilution of mouse $\mathrm{MAb}$ against acetylated alpha tubulin (ciliated cells) (Abcam, Cambridge, UK), or a 1:100 dilution of rabbit anti-cytokeratin-5 primary antibody (basal epithelial cells) (Abcam, Cambridge, UK) overnight at $4^{\circ} \mathrm{C}$. After washing, cells were exposed to either a 1:250 dilution of Alexafluor 488 goat anti-rabbit IgG (Invitrogen Ltd., Paisley, UK), a 1:250 dilution of Alexafluor 568 goat anti-mouse IgG (Invitrogen Ltd., Paisley, UK), or a 1:250 dilution of goat anti-rabbit IgG FITC at $4^{\circ} \mathrm{C}$ in the dark for $1 \mathrm{~h}$. Cells were once again washed thrice in PBS, and the membrane was then cut out using a scalpel and mounted on a microscope slide using Vectashield with DAPI (Vector Laboratories, Peterborough, UK). Negative control slides were also prepared by omitting the primary antibodies to demonstrate specificity (Fig. 4E). Fluorescent images were viewed on a Leica SP5 confocal DMI 6000 inverted microscope equipped with a krypton-argon laser as the source for the ion beam using a $40 \times$ oil immersion objective (numerical aperture 1.25). Images were captured and viewed using LAS AF (Leica) acquisition software.

Transmission electron microscopy. Cells selected for transmission electron microscopy (TEM) were fixed and processed as described by Sajjan et al. (16). Briefly, cells were fixed in universal fixative (1\% osmium tetroxide) for $1 \mathrm{~h}$ followed by dehydration in a series of graded ethanols. Cultures were infiltrated with epon resin and $80 \mathrm{~nm}$ sections cut. Sections were then mounted on copper grids and stained with 5\% uranyl acetate $/ 2.6 \%$ lead citrate and then observed under a Philips CM 100 transmission electron microscope.

Statistical Analysis. All data are expressed as mean \pm SD except for the MUC5AC ELISA where all data are expressed as mean \pm SEM. A repeated measures of ANOVA with time as a within and asthma (Yes/No) as a between subject factor was performed to analyze the MUC5AC ELISA data. A $t$ test was used to compare IL-6, IL-8, and PGE2 ELISA and cytospin data. Comparison of cytokines between groups was made using $t$ test. A $p<0.05$ indicated statistical significance.

\section{RESULTS}

Primary cell culture and establishment of the ALI. A number of different conditions such as collagen coating of the Transwell membranes, cell seeding density, different growth, and differentiation media were tested in extensive preliminary experiments (data not shown). For the optimal growth and differentiation of PBECs, the following conditions were established and followed throughout this study. PBECs were cultured on collagen coated T75 flasks and reached $\sim 80 \%$ confluence within 7 to $10 \mathrm{~d}$. Following passage onto 12-well collagen coated Transwells at a seeding density of $0.8 \times 10^{5}$ cells/transwell, cells grew to confluence within 5 to $7 \mathrm{~d}$. When viewed under a light microscope at a low magnification $(20 \times)$, the cells demonstrated a "cobblestone" arrangement that was characteristic of epithelial cells in culture. After $\sim 14 \mathrm{~d}$ of culture at ALI, mucus secretion was visible on the apical surface of the cell layer and this continued to be the case until the end of the culture period at $\mathrm{d} 28$. When viewed using a light microscope at a higher magnification $(40 \times)$, it was possible to observe cilia beating from approximately d 14 onwards.

\section{Morphologic characterization of nonasthmatic pediatric} ALI cell cultures. To assess the morphology of in vitro differentiated epithelium, cells from nonasthmatic children were fixed with $4 \%$ PFA, embedded in paraffin wax, sectioned, and stained. The presence of a number of layers of cells was observed, including ciliated cells on the apical
A

Multi-layered columnar epithelium
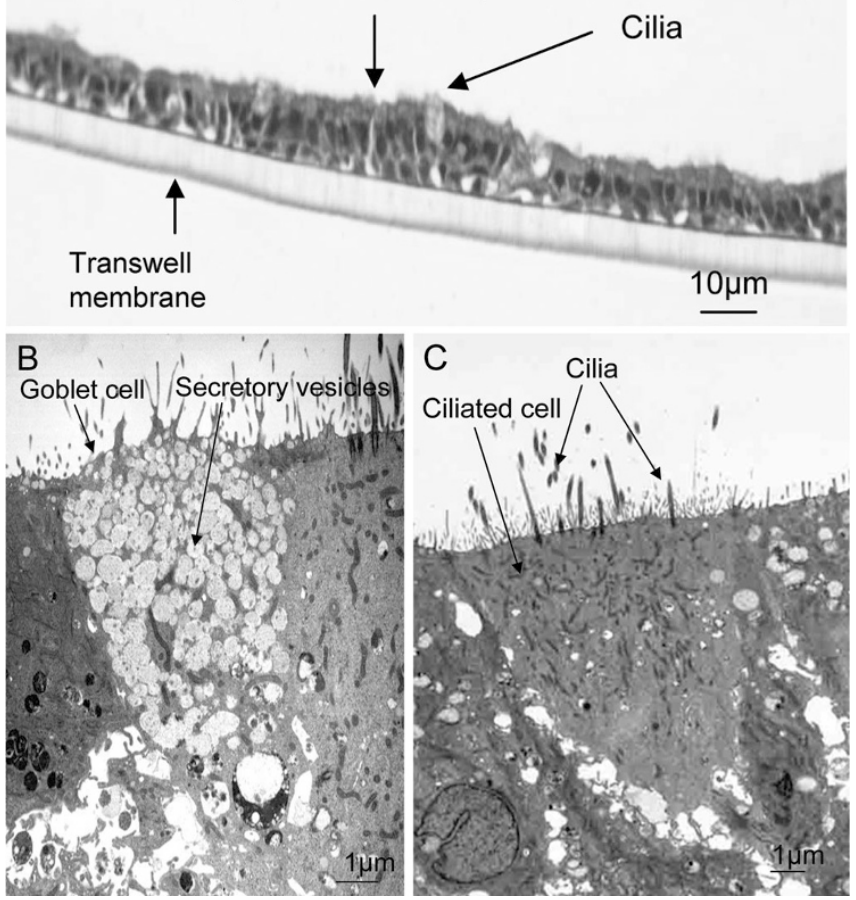

Figure 1. (A) Cross section $(5 \mu \mathrm{m})$ of PBECs cultured for $28 \mathrm{~d}$ stained with hematoxylin and eosin. Multilayered epithelial cells can be clearly seen. Cilia can be observed on the apical surface $(\times 40)$. (B) Transmission electron micrograph of showing a columnar goblet cell containing electron-lucent secretory granules $(\times 12,000)$. $(C)$ Transmission electron micrograph of a ciliated cell at the apical surface of the epithelium $(\times 9000)$.

surface of the epithelial cell layer (Fig. 1A). To further confirm the cellular identity of pediatric bronchial epithelial ALI cultures, TEM was used. This ultrastructural analysis revealed the presence of goblet cells containing electron-lucent secretory granules associated with mucus secretion at the apical surface (Fig. $1 B$ ). Figure $1 C$ shows a representative electron micrograph of a ciliated cell at the apical surface of the epithelium. The observed presence of a multilayered epithelium containing ciliated and goblet cells demonstrated strongly the complete differentiation of PBECs into a welldifferentiated and functioning epithelium.

MUC5AC expression and quantification throughout the culture. RT-PCR was performed to confirm the production of MUC5AC mRNA during the 28-d culture period at ALI. In all nonasthmatic subjects, it was evident that MUC5AC mRNA production was underway by d 14 of ALI culture (Fig. 2A). MUC5AC mRNA continued to be produced throughout the culture period. Apical secretion of mucus by WD-PBECs was measured by MUC5AC ELISA (Fig. 2B). The cells produced small amounts of mucin by $\mathrm{d} 7$ but significantly more by $\mathrm{d} 28$ when compared with $\mathrm{d} 7(p<0.01)$. This confirmed that MUC5AC secreting goblet cells were present and functional in the epithelium.

Comparison of asthmatic and nonasthmatic pediatric epithelium using the ALI culture model. TEER was measured on $\mathrm{d} 7,14,21$, and 28 in both study groups. The TEER at each time point during the study was measured as between 500 and 
$700 \Omega \cdot \mathrm{cm}^{2}$, which is similar to what others have reported as indicating tight junction formation (17). Comparing nonasthmatic and asthmatic log transformed TEER during the 28-d culture period, there was no significant difference observed at any time point (Fig. 3A). Cytospins prepared from d 28 cultures demonstrated morphologic phenotypes, in terms of goblet cell (Fig. 3B) and ciliated cell (Fig. 3C) content, which was reproducibly characteristic of either asthmatic or nonasthmatic origins. We found that cultures developed from asthmatic children had $36.7 \%$ goblet cells and $15.6 \%$ ciliated cells compared with $18.8 \%$ goblet cells and $23.0 \%$ ciliated cells in nonasthmatics ( $p<0.05$ and $p<0.05$, respectively). We confirmed this observation using immunofluorescent staining for MUC5AC and acetylated alpha tubulin in conjunction with confocal microscopy (Fig. 4). Nonasthmatic epithelium exhibited a limited number of MUC5AC positive goblet cells
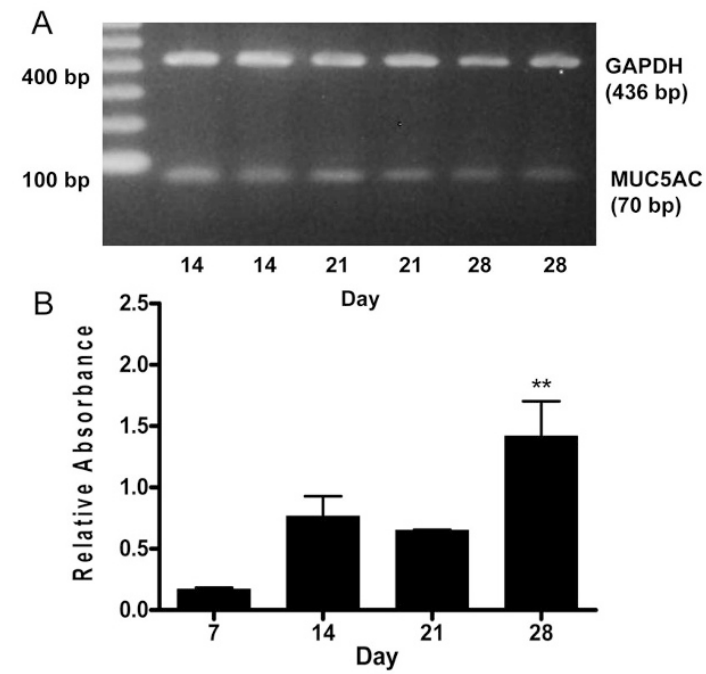

Figure 2. (A) Expression of MUC5AC mRNA measured on d 14, 21, and 28 of ALI culture using RT-PCR and gel electrophoresis. Housekeeping gene used was GAPDH (436 bp). MUC5AC showed expression at $70 \mathrm{bp}$. In each gel, a 100-bp ladder was used for reference. (B) MUC5AC ELISA of apical washings taken during a 28 -d culture period at ALI. Average relative absorbance of apical washings measured for MUC5AC secretion. Values expressed are mean \pm SEM for each patient. ${ }^{* *} p<0.01$ for comparison of average values at $\mathrm{d} 28$ and baseline $\mathrm{d} 7$. (stained in green) (Fig. $4 A$ and $B$ ) compared with asthmatic cultures (Fig. $4 C$ and $D$ ), which clearly have a larger number of goblet cells within their epithelium. In addition, we also stained for basal cells alongside either goblet or ciliated cells in both nonasthmatic and asthmatic cultures to demonstrate that all three cell types were present within the differentiated epithelium (Figs. 5 and 6). Comparisons between cytokine ELISA's for proinflammatory and antiinflammatory cytokines showed no significant differences between the quantities of IL-8 $(28,268 \pm 16,539 \mathrm{pg} / \mathrm{mL} ; 22,880 \pm 18,881 \mathrm{pg} / \mathrm{mL})$, IL6 $(113.4 \pm 47.0 \mathrm{pg} / \mathrm{mL} ; 70.3 \pm 165.9 \mathrm{pg} / \mathrm{mL})$, or PGE2 $(79.4 \pm$ $12.8 \mathrm{pg} / \mathrm{mL} ; 125.4 \pm 88.22 \mathrm{pg} / \mathrm{mL}$ ) being produced by either asthmatic or nonasthmatic cultures, respectively (graphs not shown). Furthermore, there was no significant difference in the amount of MUC5AC secreted onto the apical surface of the asthmatic and nonasthmatic cultures (data not shown). There was no significant difference in the overall total cell number between both study groups (data not shown).

\section{DISCUSSION}

In this study, we have shown that it is possible to grow PBECs and differentiate them into a functional epithelium in an ALI system. Progressive cellular differentiation was observed during the culture period, with the development of basal, ciliated, and goblet cells being confirmed at the conclusion of the culture period using extensive histologic investigational techniques. Differentiated cells could be successfully cultured for $28 \mathrm{~d}$ at ALI, which has been reported to be the optimal study time for primary human epithelial cells after confluence (18). It was possible to observe cilia beating from d 14 of ALI culture along with the visible production of mucus at the apical layer. Expression of the major mucus-forming mucin, MUC5AC, was easily detected at the mRNA level, as well as by mucin ELISA, as early as d 7, although significantly increased amounts were demonstrated by ELISA at d 28 .

Having established a culture model that better mimics the bronchial epithelium in vivo, we assessed the potential morphologic and functional differences that were thought to exist between nonasthmatic and asthmatic bronchial epithelium. Our study has clearly demonstrated intrinsic morphologic
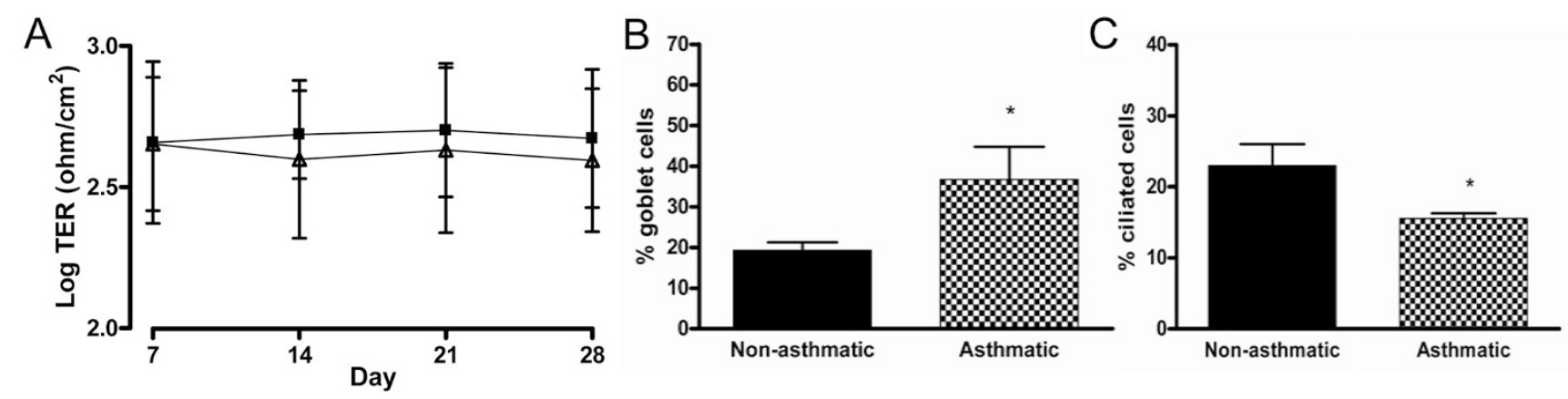

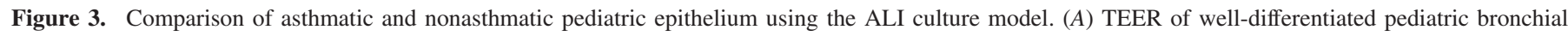

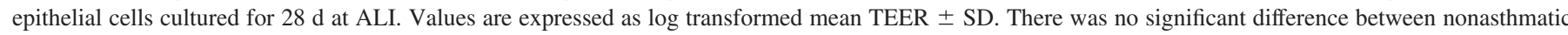

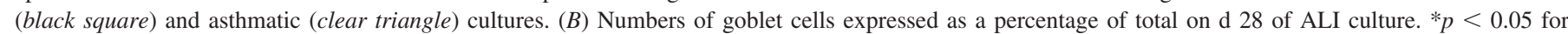

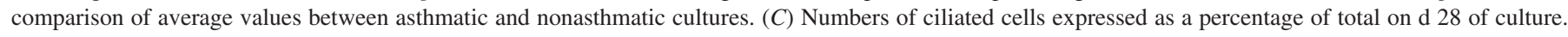
$* p<0.05$ for comparison of average values between asthmatic and nonasthmatic cultures. 

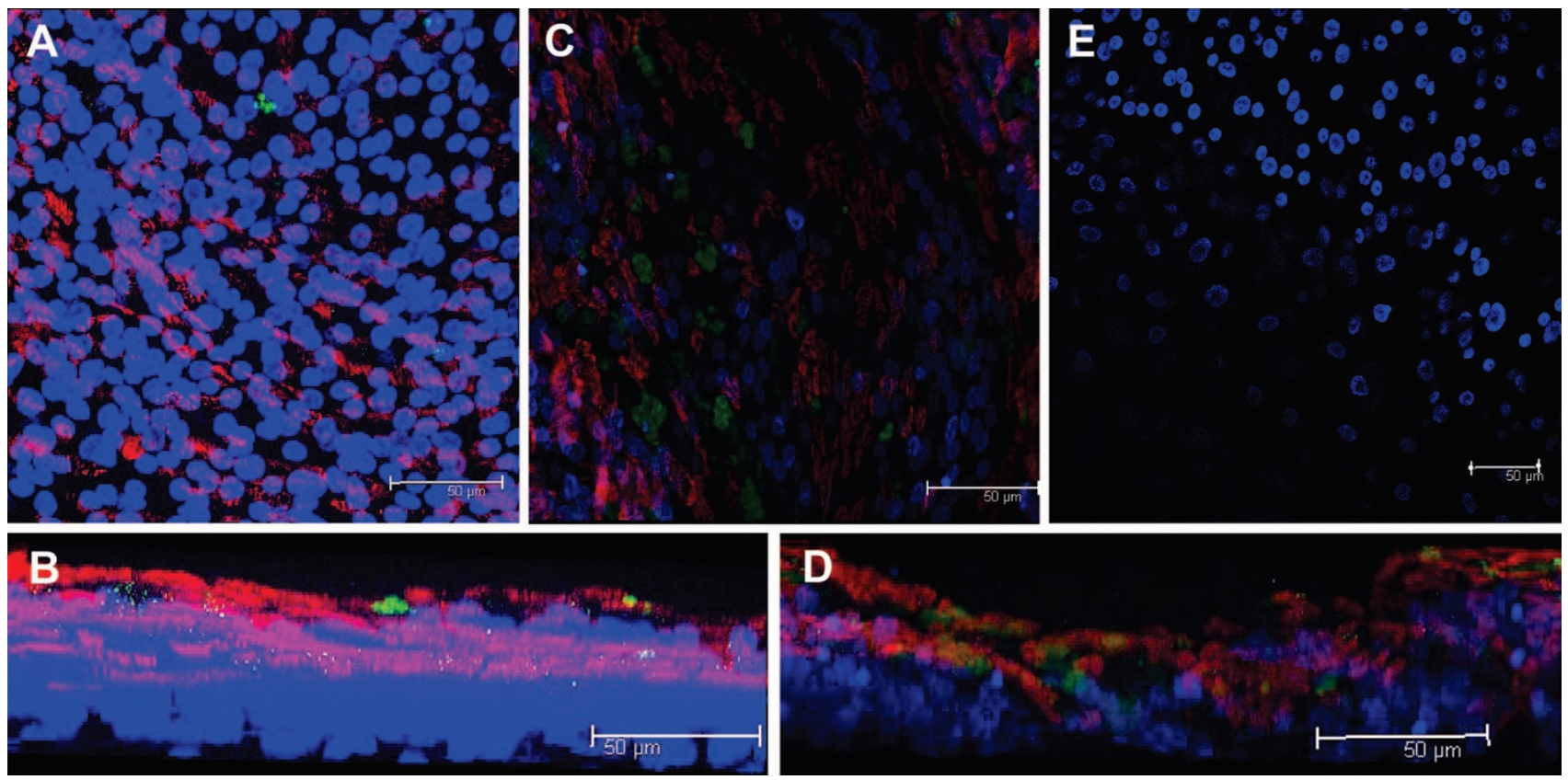

Figure 4. Immunofluorescence and confocal microscopy of asthmatic and nonasthmatic cultures. Representative image of the $(A)$ apical surface and $(B)$ z-stack of a nonasthmatic pediatric bronchial epithelium at d 28 cultures at ALI. Goblet cell presence indicated by green staining and ciliated cell presence indicated by red staining, with nuclei indicated by blue staining with DAPI $(\times 40)$. Representative image of the $(C)$ apical surface and $(D)$ z-stack of an asthmatic pediatric bronchial epithelium at d 28 culture at ALI. Goblet cell presence indicated by green staining and ciliated cell presence indicated by red staining, with nuclei indicated by blue staining with DAPI $(\times 40)$. It is possible to see a higher number of MUC5AC positive goblet cells in the asthmatic cultures. $(E)$ Negative control image of pediatric bronchial epithelium with primary antibody omitted $(\times 40)$.

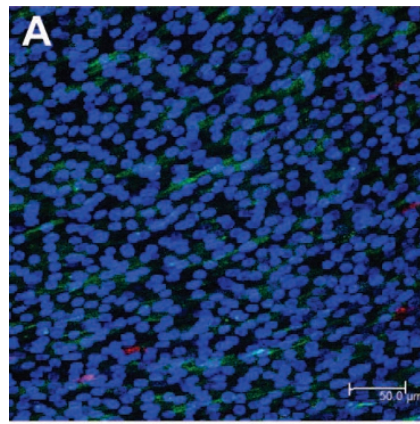

\section{B}

Figure 5. Immunofluorescence and confocal microscopy of nonasthmatic cultures. Representative maximum intensity images of the $(A)$ apical surface and $(B)$ z-stack of nonasthmatic pediatric bronchial epithelium stained for goblet cells $($ red $)$ and basal epithelial cells (green) at d 28 culture $(\times 40)$. Representative maximum intensity images of the $(C)$ apical and $(D)$ z-stack of nonasthmatic pediatric bronchial epithelium stained for ciliated cells ( $r e d$ ) and basal cells (green) at d 28 culture. Nuclei in all images stained blue with DAPI (×40).

differences between bronchial epithelial cells from asthmatic and nonasthmatic children. Asthmatic bronchial epithelial cell cultures contain higher numbers of mucus-secreting goblet cells and lower numbers of ciliated cells when compared with nonasthmatic cultures under identical unstimulated culture conditions. Our model of pediatric bronchial epithelium has demonstrated in asthmatic cultures, characteristics that are seen in vivo, in the manifestation of goblet cell hyperplasia (19). The increased number of goblet cells measured in asthmatic in vitro epithelia, however, was not reflected in an increased secretion of MUC5AC in the apical lumen. It is
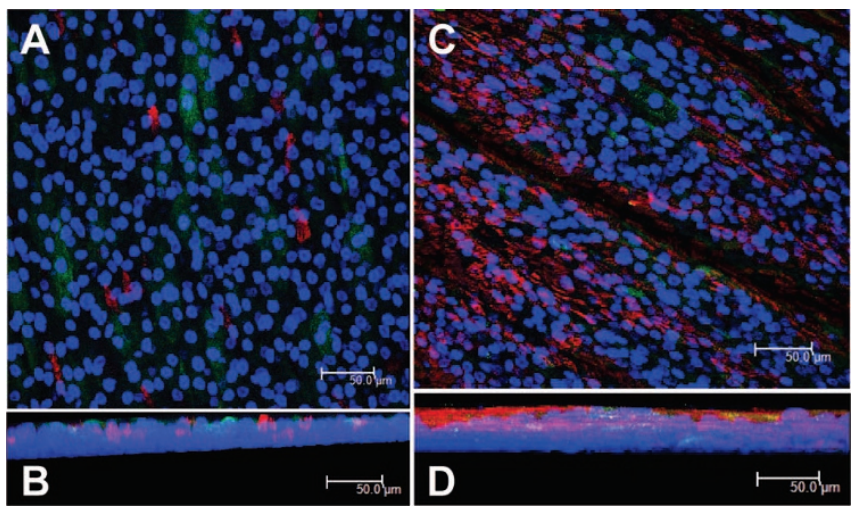

Figure 6. Immunofluorescence and confocal microscopy of asthmatic cultures. Representative maximum intensity images of the $(A)$ apical surface and $(B)$ z-stack of asthmatic pediatric bronchial epithelium stained for goblet cells (red) and basal epithelial cells (green) at d 28 culture $(\times 40)$. Representative maximum intensity images of the $(C)$ apical and $(D)$ z-stack of asthmatic pediatric bronchial epithelium stained for ciliated cells (red) and basal cells (green) at d 28 culture. Nuclei in all images stained blue with DAPI $(\times 40)$.

possible that additional stimuli are necessary to induce mucus secretion from the goblet cells, and this is something our current studies are focusing on, including investigating the mechanism(s) behind this goblet cell hyperplasia. We believe that our culture model is advantageous in that it better resembles the epithelium in vivo, having displayed phenotypic characteristics associated with pediatric asthma.

Interestingly, Kicic et al. (7) reported differences between pediatric asthmatic and nonasthmatic bronchial epithelial cells, including substantially increased expression of IL-6 and PGE2 in asthmatic submerged cultures. This may be due to their submerged model representing a more inflamed model as 
the culture media contains stimulants on a surface normally exposed to air. In our well-differentiated model, in contrast, we found no significant difference in IL-6 or PGE2 levels between asthmatic and nonasthmatic pediatric epithelial cells. However, our data on spontaneous IL-8 secretion concurred with Kicic et al., who also found there to be no significant difference in secreted levels of IL- 8 between asthmatic and nonasthmatic cells on d 28 of culture. These results highlight differences in epithelial function dependent on the type of culture system used. As our WD-PBEC cultures closely resemble bronchial epithelium in vivo, we suggest that our data provides a better reflection of the scenario within both asthmatic and nonasthmatic airways compared with monolayer cultures.

In conclusion, our well-differentiated model of pediatric epithelium demonstrated intrinsic differences between asthmatic and nonasthmatic bronchial epithelium under identical unstimulated culture conditions that are characteristic of phenotypes seen in asthmatic airways (19). This suggests an inherent difference between asthmatic and nonasthmatic pediatric bronchial epithelium. As such, it provides us with a novel and physiologically relevant platform to elucidate pathophysiological mechanisms associated with disease states such as asthma and, ultimately, novel therapeutics.

Acknowledgments. We thank the anesthetists at the Royal Belfast Hospital for Sick Children, parents, and children for facilitating this study. Prof. Madeleine Ennis, Dr. Vanessa Brown, and Dr. Scott McKeown provided valuable critical comments on this manuscript.

\section{REFERENCES}

1. Holgate ST, Lackie P, Wilson S, Roche W, Davies D 2000 Bronchial epithelium as a key regulator of airway allergen sensitization and remodeling in asthma. Am J Respir Crit Care Med 162:S113-S117
2. Knight DA, Holgate ST 2003 The airway epithelium: structural and functional properties in health and disease. Respirology 8:432-466

3. van den Toorn LM, Overbeek SE, de Jongste JC, Leman K, Hoogsteden HC, Prins JB 2001 Airway inflammation is present during clinical remission of atopic asthma. Am J Respir Crit Care Med 164:2107-2113

4. Cutz E, Levison H, Cooper DM 2002 Ultrastructure of airways in children with asthma. Histopathology 41:22-36

5. Pohunek P, Warner JO, Turzikova J, Kudrmann J, Roche WR 2005 Markers of eosinophilic inflammation and tissue re-modelling in children before clinically diagnosed bronchial asthma. Pediatr Allergy Immunol 16:43-51

6. Doherty GM, Christie SN, Skibinski G, Puddicombe SM, Warke TJ, de Courcey F, Cross AL, Lyons JD, Ennis M, Shields MD, Heaney LG 2003 Non-bronchoscopic sampling and culture of bronchial epithelial cells in children. Clin Exp Allergy 33:1221-1225

7. Kicic A, Sutanto EN, Stevens PT, Knight DA, Stick SM 2006 Intrinsic biochemical and functional differences in bronchial epithelial cells of children with asthma. Am J Respir Crit Care Med 174:1110-1118

8. Gray TE, Guzman K, Davis CW, Abdullah LH, Nettesheim P 1996 Mucociliary differentiation of serially passaged normal human tracheobronchial epithelial cells. Am J Respir Cell Mol Biol 14:104-112

9. Fulcher ML, Gabriel S, Burns KA, Yankaskas JR, Randell SH 2005 Welldifferentiated human airway epithelial cell cultures. Methods Mol Med 107:183-206

10. Becker MN, Sauer MS, Muhlebach MS, Hirsch AJ, Wu Q, Verghese MW, Randell SH 2004 Cytokine secretion by cystic fibrosis airway epithelial cells. Am J Respir Crit Care Med 169:645-653

11. Pickles RJ, McCarty D, Matsui H, Hart PJ, Randell SH, Boucher RC 1998 Limited entry of adenovirus vectors into well-differentiated airway epithelium is responsible for inefficient gene transfer. J Virol 72:6014-6023

12. Zhang L, Peeples ME, Boucher RC, Collins PL, Pickles RJ 2002 Respiratory syncytial virus infection of human airway epithelial cells is polarized, specific to ciliated cells, and without obvious cytopathology. J Virol 76:5654-5666

13. Stevenson EC, Turner G, Heaney LG, Schock BC, Taylor R, Gallagher T, Ennis M, Shields MD 1997 Bronchoalveolar lavage findings suggest two different forms of childhood asthma. Clin Exp Allergy 27:1027-1035

14. Takeyama K, Dabbagh K, Lee HM, Agusti C, Lausier JA, Ueki IF, Grattan KM, Nadel JA 1999 Epidermal growth factor system regulates mucin production in airways. Proc Natl Acad Sci USA 96:3081-3086

15. Yoon JH, Gray T, Guzman K, Koo JS, Nettesheim P 1997 Regulation of the secretory phenotype of human airway epithelium by retinoic acid, triiodothyronine, and extracellular matrix. Am J Respir Cell Mol Biol 16:724-731

16. Sajjan U, Ackerley C, Forstner J 2002 Interaction of cblA/adhesin-positive Burk holderia cepacia with squamous epithelium. Cell Microbiol 4:73-86

17. Lin H, Li H, Cho HJ, Bian S, Roh HJ, Lee MK, Kim JS, Chung SJ, Shim CK, Kim DD 2007 Air-liquid interface (ALI) culture of human bronchial epithelial cell monolayers as an in vitro model for airway drug transport studies. J Pharm Sci 96:341-350

18. Yoon JH, Moon HJ, Seong JK, Kim CH, Lee JJ, Choi JY, Song MS, Kim SH 2002 Mucociliary differentiation according to time in human nasal epithelial cell culture. Differentiation 70:77-83

19. Sumi Y, Hamid Q 2007 Airway remodeling in asthma. Allergol Int 56:341-348 